\title{
A Deduction from Dirac Sea Model
}

\author{
Jianguo Bian1 $1 *$ and Jiahui Wang $^{2}$ \\ 1 Institute of High Energy Physics, Beijing 100049, China \\ 2 China Agricultural University, Beijing 100083, China
}

(Dated: September 15, 2021)

\begin{abstract}
Whether the Dirac sea model is right is verifiable. Assuming the Dirac sea is a physical reality, we have one imagination that a negative energy particle(s) in the sea and a usual positive energy particle(s) will form a neutral atom. The features of the atom can be studied using the nonrelativistic reduction of the Bethe-Salpeter equation, especially for a two body system. This work is dedicated to discuss the bound state of spin-0 and spin- $\frac{1}{2}$ constituents. The study shows that an atom consisting of a negative energy particle $\left(e^{-}\right.$or $\left.h c\right)$ and a positive energy particle $(u \bar{d}$ or $h c)$ has some features of a neutrino, such as spin, lepton number and oscillation. One deduction is that if the atom is a real particle, an atom $X$ consisting of two negative energy particles and a nucleus with double charges exists in nature, which can be observed in Strangeonium, Charmonium and Bottomonium decays $[s \bar{s}],[c \bar{c}]$ and $[b \bar{b}] \rightarrow X \pi^{-} \pi^{-} e^{+} e^{+}+h c$ with $X$ invisible at KLOE, CLEOc, BESIII, Belle and BaBar.

PACS numbers:
\end{abstract}

Keywords: Dirac Sea, negative energy electron, nonrelativistic reduction of the Bethe-Salpeter equation, exotic atom, two body system, observation proposal

*Electronic address: bianjg@mail.ihep.ac.cn 
The Dirac sea ${ }^{[1]}$ is a theoretical model of the vacuum as an infinite sea of particles with negative energy. It was first postulated by the British physicist Paul Dirac in 1930 to explain the anomalous negative-energy quantum states predicted by the Dirac equation, an extension of the Schrödinger equation ${ }^{[2]}$ for relativistic electrons. The positron, the antimatter counterpart of the electron, was originally conceived of as a hole in the Dirac sea, well before its experimental discovery in 1932. Despite its success, the idea of the Dirac sea tends not to strike people as very elegant. The development of quantum of filed in the 1930s reformulate the Dirac equation in a way that treats the positron as a real particles rather than the absence of a particle and make the vacuum the state in which no particles exist instead of an infinite sea of particles. Therefore a question appears whether the idea of the Dirac sea is completely wrong and how to verify it? For instance, for the process $\gamma \rightarrow e^{+} e^{-}$, the description of the quantum of filed is that a gamma converts into a pair of electron and positron, but the language of the Dirac sea model is that a gamma hits a nagetive energy electron; the electron excites into an positive energy state and an positron appears simutaniously as a "hole". The two pictures are quite different. Either of the two is right? The aim of this article is to propose an observation scheme to verify the correction of the Dirac sea. We assume the idea of the Dirac sea is right, extend it to including negative energy positrons and the other leptons and use the nonrelativistic reduction of the Bethe-Salpeter equation ${ }^{[3]}$ to investigate a possibility that a usual positive energy particle $(u \bar{d}$ or $h c)$ and a negative energy particle $\left(e^{-}\right.$or $\left.h c\right)$ in the Dirac sea will form a neutral atom and study special properties of this kind of atoms. The article is organized as follow. The nonrelativistic reduction of the Bethe-Salpeter equation about a two body system of spin- 0 and spin- $\frac{1}{2}$ constituents is presented in paragraphs 2 . The detailed steps to solve the equation with $E<0$ and $E>0$ are described in paragraphs 3 and 4, respectively. The 
physical information is extracted from the solution to the equation in paragraph 5 . The final paragraph arrives at a summary.

The nonrelativistic reduction of the Bethe-Salpeter equation for a charge-anticharge two body system of spin-0 and spin- $\frac{1}{2}$ positive energy constituents $\left(E_{s}=\sqrt{q_{s}^{2}+M_{s}^{2}}, E_{f}^{\prime}=\right.$ $\left.\sqrt{q_{s}^{2}+m_{f}^{2}}\right)$ through the single-photon and "seagull" interaction shown in Fig. (1) has been given in Ref.[4],

$$
E^{\prime} \psi(\mathbf{r})=-\left(\frac{1}{2 M_{s}}+\frac{1}{2 m_{f}}\right) \nabla^{2} \psi(\mathbf{r})-\frac{q^{2}}{r} \psi(\mathbf{r})-\frac{7 q^{4}}{4 M_{s}} \frac{1}{r^{2}} \psi(\mathbf{r})
$$

where $M_{s}$ and $q$ are the mass and charge of the spin-0 particle and $m_{f}$ and $-q$ are the mass and charge of the spin- $\frac{1}{2}$ particle, the nonrelativistic energy $E^{\prime}$ is related to the relativistic energy $E_{r}^{\prime}$ by $E_{r}^{\prime}=M_{s}+m_{f}+E^{\prime}$. From Eq. (11) it is straightforward to make the nonrelativistic reduction of the Bethe-Salpeter equation for a charge-anticharge two body system in which the spin-0 constituent is a positive energy particle while the spin- $\frac{1}{2}$ constituent is a negative energy particle $E_{f}=-\sqrt{q_{f}^{2}+m_{f}^{2}}$,

$$
E \psi(\mathbf{r})=-\left(\frac{1}{2 M_{s}}-\frac{1}{2 m_{f}}\right) \nabla^{2} \psi(\mathbf{r})-\frac{q^{2}}{r} \psi(\mathbf{r})-\frac{7 q^{4}}{4 M_{s}} \frac{1}{r^{2}} \psi(\mathbf{r}),
$$

where the nonrelativistic energy $E$ is related to the relativistic energy $E_{r}$ by

$$
E_{r}=M_{s}-m_{f}+E \geq 0
$$

It is worth emphasizing that in Eq. (2), $M_{s}$ is a physical state, while $m_{f}$ is a vacuum state. One considers that a vacuum state can not be observed as a physical state directly, but there are interactions between physical states and vacuum states. In Eq. (3), $-m_{f}$ alone 
does not represent a negative mass particle, instead $-m_{f}$ and $-\frac{q_{f}^{2}}{2 m_{f}}$ together describe the nonrelativistic reduction of the relativistic energy of a negative energy particle $E_{f}=$ $-\sqrt{q_{f}^{2}+m_{f}^{2}}$. In spherical polar coordinates

$$
\psi(\mathbf{r})=R(r) \Theta(\theta) \Phi(\phi) .
$$

In this work, only radial wave function $R(r)$ is worth discussing,

$$
\frac{1}{r^{2}} \frac{d}{d r}\left(r^{2} \frac{d R}{d r}\right)-\left[\frac{2 \mu}{\hbar^{2}}\left(E+\frac{q^{2}}{r}+\frac{7 q^{4}}{4 M_{s}} \frac{1}{r^{2}}\right)+\frac{l(l+1)}{r^{2}}\right] R=0 .
$$

Let $R(r)=\frac{u(r)}{r}$, one has

$$
\frac{d^{2} u}{d r^{2}}-\left[\frac{2 \mu}{\hbar^{2}}\left(E+\frac{q^{2}}{r}+\frac{7 q^{4}}{4 M_{s}} \frac{1}{r^{2}}\right)+\frac{l(l+1)}{r^{2}}\right] u=0,
$$

where the reduced mass $\mu=\frac{m_{f} M_{s}}{M_{s}-m_{f}}$.

For $E<0$, one has

$$
\frac{d^{2} u}{d r^{2}}+\left[\frac{2 \mu}{\hbar^{2}}\left(|E|-\frac{q^{2}}{r}-\frac{7 q^{4}}{4 M_{s}} \frac{1}{r^{2}}\right)-\frac{l(l+1)}{r^{2}}\right] u=0 .
$$

Eq. (7) has the same form of the Schrödinger equation for two body scattering in repulsive fields ${ }^{[5]}$

$$
\frac{d^{2} u}{d r^{2}}+\left[\frac{2 \mu^{\prime}}{\hbar^{2}}\left(E-\frac{Z q^{2}}{r}\right)-\frac{l(l+1)}{r^{2}}\right] u=0
$$

where $E>0$ and the reduced mass $\mu^{\prime}=m M /(M+m)$. Let $\alpha=\left(\frac{8 \mu|E|}{\hbar^{2}}\right)^{\frac{1}{2}}, \eta=$ 
$\frac{q^{2}}{\hbar}\left(\frac{\mu}{2|E|}\right)^{\frac{1}{2}}$ and $\rho=\frac{\alpha r}{2}, \gamma=\frac{7 q^{4}}{\hbar^{2}} \frac{\mu}{2 M_{s}}$, Eq. (77) becomes

$$
\frac{d^{2} u}{d \rho^{2}}+\left[1-\frac{2 \eta}{\rho}-\frac{\gamma}{\rho^{2}}-\frac{l(l+1)}{\rho^{2}}\right] u=0
$$

i.e.

$$
\frac{d^{2} u}{d \rho^{2}}+\left[1-\frac{2 \eta}{\rho}+\frac{\frac{1}{4}-L^{2}}{\rho^{2}}\right] u=0
$$

where

$$
L=\sqrt{\left(l+\frac{1}{2}\right)^{2}+\gamma}
$$

Eq. (9) is called the Coulomb wave equation ${ }^{[5]}$, which has two independent solutions

$$
u(\eta, \rho)=C_{1} F_{L}(\eta, \rho)+C_{2} G_{L}(\eta, \rho) \quad\left(C_{1}, C_{2} \text { constants }\right)
$$

where $F_{L}(\eta, \rho)$ is the regular Coulomb wave function and $G_{L}(\eta, \rho)$ is the irregular Coulomb wave function. The asymptotic forms of the Coulomb wave functions can be obtained from the theory of the confluent hypergeometric function.

$$
\begin{gathered}
F_{L}(\eta, \rho)=C_{L}(\eta) \rho^{L+1}[1+O(\rho)] \quad \text { as } \rho \rightarrow 0 . \\
F_{L}(\eta, \rho)=\sin \left(\rho-\eta \ln (2 \rho)-\frac{1}{2} L \pi+\sigma_{L}(\eta)\right)+O\left(\rho^{-1}\right) \quad \text { as } \rho \rightarrow \infty,
\end{gathered}
$$


where $\sigma_{L}(\eta)=\arg \Gamma(L+1+i \eta)$.

$$
G_{L}(\eta, \rho)=O\left(\rho^{-L}\right) \quad \text { as } \rho \rightarrow 0 .
$$

$$
G_{L}(\eta, \rho)=\cos \left(\rho-\eta \ln (2 \rho)-\frac{1}{2} L \pi+\sigma_{L}(\eta)\right)+O\left(\rho^{-1}\right) \quad \text { as } \rho \rightarrow \infty .
$$

From Eqs. (10, 14), it follows that $\int_{0}^{\infty} r^{2} \frac{G_{L}^{2}(\eta, r)}{r^{2}} d r$ is not integrable for any $l$. From Eqs.(13, 15), it is noted that $F_{L}(\eta, \rho)$ and $G_{L}(\eta, \rho)$ describe the scattered particle radial wave functions of Eq. (6).

For $E>0$, Eq. (66) becomes

$$
\frac{d^{2} u}{d \rho^{2}}+\left[-1-\frac{2 \eta}{\rho}+\frac{\frac{1}{4}-L^{2}}{\rho^{2}}\right] u=0 .
$$

Let

$$
u=e^{-\rho} f(\rho),
$$

$f(\rho)$ satisfies

$$
f^{\prime \prime}(\rho)-2 f^{\prime}(\rho)+\left[-\frac{2 \eta}{\rho}+\frac{\frac{1}{4}-L^{2}}{\rho^{2}}\right] f(\rho)=0 .
$$

Let

$$
f(\rho)=\sum_{n=0}^{\infty} a_{n} \rho^{n+s}
$$


and substitute it to eq. (17), one has the relation

$$
a_{n+1}=\frac{2 \eta+2(n+s)}{(n+1+s)(n+s)-\gamma-l(l+1)} a_{n} .
$$

Due to $a_{0} \neq 0$, one has

$$
s(s-1)=l(l+1)+\gamma
$$

The s solution is

$$
s=\frac{1}{2} \pm \sqrt{\left(l+\frac{1}{2}\right)^{2}+\gamma} .
$$

From eqs. (19, 20), it is required

$$
2 s>-1 \text {. }
$$

and

$$
\eta+n+s \leq 0
$$

i.e.

$$
s \leq 0,
$$


due to $\eta \geq 0$ and $n \geq 0$ so that $\int_{0}^{\infty} r^{2} R^{2}(r) d r$ is integrable. From eq. (23) , one has

$$
l(l+1)+\gamma<\frac{3}{4}
$$

From eq. (25), one has

$$
l(l+1)+\gamma \geq 0
$$

Combining eqs. (26, 27), one has

$$
l=0
$$

and an exact solution of $f(\rho)$

$$
\begin{gathered}
s=\frac{1}{2}-\sqrt{\frac{1}{4}+\gamma}, \\
\eta=-s=\sqrt{\frac{1}{4}+\gamma}-\frac{1}{2}, \\
a_{n}=0, n=1,2, \ldots
\end{gathered}
$$

It is easy to check if the spin- 0 constituent is a $\pi^{ \pm}$and the spin- $\frac{1}{2}$ is an $e^{\mp}$ or $\mu^{\mp}, 0<\gamma<\frac{3}{4}$.

Now, we turn to extract the physical information from above discussion. Eq. (66) has a bound state solution described by Eqs. (28, 31) for the energy $E=\frac{q^{2}}{\hbar} \frac{\mu}{2 \eta^{2}}$ and only the orbital quantum number $l=0$. Eq. (6) also has a scattering state solution for the continuum 
$-M_{s}+m_{f}<E<0$, here the lower limit is given by Eq. (3). Therefore the bound state with energy $E>0$ will transit to the scattering state with energy $E<0$, then the positive energy constituent and another negative energy particle in the Dirac sea will form a new bound state and the new bound state will transit. The process of formation and transition cycles at intervals of

$$
\Delta t \geq \frac{\hbar}{2\left(\frac{q^{2}}{\hbar} \frac{\mu}{2 \eta^{2}}+M_{s}-m_{f}\right)}
$$

based on the uncertainty principle $\Delta t \Delta E \geq \frac{\hbar}{2}$. If the positive energy particle consists of $u \bar{d}$ and the negative energy particle is an electron, $u \bar{d}$ and $e^{-}$will form an atom we call $X\left(e^{-} u \bar{d}\right)$. The atom has some features of the neutrino $\nu_{e}$. The spin quantum is $\frac{1}{2}$. The lepton number is 1. $X\left(e^{-} u \bar{d}\right)$ will transit to the scattering state and then a new atom $X\left(e^{-} u \bar{d}\right)$ or $X\left(\mu^{-} u \bar{d}\right)$ will form as a neutrino oscillates. However, in this simple work, the mass of the bound state can not be obtained due to the non-relativistic reduction. If $X\left(e^{-}\right)$is a real particle, one deduction is that four quarks $u \bar{d} u \bar{d}$ and two negative energy electrons $e^{-} e^{-}$can also form an atom we call $X\left(2\left(e^{-} u \bar{d}\right)\right)$. The $X\left(2\left(e^{-} u \bar{d}\right)\right)$ can be observed in Strangeonium, Charmonium and Bottomonium decays $[s \bar{s}],[c \bar{c}]$ and $[b \bar{b}] \rightarrow X\left(2\left(e^{-} u \bar{d}\right)\right) \pi^{-} \pi^{-} e^{+} e^{+}+h c$ with $X\left(2\left(e^{-} u \bar{d}\right)\right)$ and $h c$ invisible at $\mathrm{KLOE}^{[6]}, \mathrm{CLEOc}^{[7}$, BESIII ${ }^{[8]}$, Belle ${ }^{[9]}$ and BaBar ${ }^{[10]}$.

In summary, the Dirac sea is verifiable. If the Dirac sea is a physics reality, the process $\gamma \rightarrow e^{+} e^{-}$cannot be lonely observable. This work shows that $u \bar{d}$ and a negative energy electron $e^{-}$will form an atom $X\left(e^{-} u \bar{d}\right)$ with the lepton number equal to 1 and $u \bar{d} u \bar{d}$ and two negative energy electrons $e^{-} e^{-}$will form another atom $X\left(2\left(e^{-} u \bar{d}\right)\right)$ with the lepton number equal to 2 . The former is hard to distinguish from a neutrino while the latter can be observed exclusively. One should pay attention to that in contrast to those well-known 
normal atoms which consist of all positive energy components and have long life-time, this kind of atoms are unstable for they oscillate from a state which has electron components to another state which has muon components. If the $X\left(2\left(e^{-} u \bar{d}\right)\right)$ is not observed, we should doubt the correction of the Dirac sea. 
FIG. 1: Feynman diagrams for (a) single-photon exchange and (b) the "seagull" interaction. The solid, dashed and wavy lines represent a spin- $\frac{1}{2}$ fermion, a spin-0 boson and a photon, respectively. 
[1] Dirac Pam, Quantized Singularities In The Electromagnetic Fields (1931).

[2] Schrödinger, E., Physical Review 28 (6), 1049 (1926).

[3] E.E. Salpeter and H.A. Bethe, Phys. Rev. 84, 1232 (1951).

[4] G. Bruce Mainland, arXiv:hep-th/0005058.

[5] M. Abramowitz and H. A. Antosiewicz, Phys. Rev. 96, 75 (1954).

[6] The KLOE Collaboration, LNF-92/019(IR), (1992).

[7] CLEO-c/CESR-c Taskforces \& CLEO-cCollaboration, Cornell University LEPPRe-port No. CLNS0 1/1742 (2001) (unpublished).

[8] BES Collaboration, Int.J.Mod.Phys.A24, 9 (2009).

[9] S. Kurokawa and E. Kikutani, Nucl. Instr. and. Meth. A499, 1 (2003).

[10] BABAR Collaboration, Nucl. Instrum. Meth. A479, 1 (2001). 
This figure "coulombseagull.JPG" is available in "JPG" format from: http://arxiv.org/ps/1210.8357v5 